tions of the plane. Every dihedral rotation group can be represented as a subtraction and division group, and the direct interpretation of these operations is always a dihedral rotation group. The operations may be resolved, when complex numbers are employed, so as to lead to other interesting groups.

F. N. CoLE, Secretary.

\title{
THE FUNDAMENTAL CONCEPTIONS AND METHODS OF MATHEMATICS.
}

\author{
ADDRESS DELIVERED BEFORE THE DEPARTMENT OF MATHE- \\ MATICS OF THE INTERNATIONAL CONGRESS OF ARTS \\ AND SCIENCE, ST. LOUIS, SEPTEMBER 20, 1904.
}

BY PROFESSOR MAXIME BÔCHER.

\section{Old and New Definitions of Mathematics.}

I am going to ask you to spend a few minutes with me in considering the question : what is mathematics? In doing this I do not propose to lay down dogmatically a precise definition; but rather, after having pointed out the inadequacy of traditional views, to determine what characteristics are common to the most varied parts of mathematics but are not shared by other sciences, and to show how this opens the way to two or three definitions of mathematics, any one of which is fairly satisfactory. Although this is, after all, merely a discussion of the meaning to be attached to a name, $I$ do not think that it is unfruitful, since its aim is to bring unity into the fundamental conceptions of the science with which we are concerned. If any of you, however, should regard such a discussion of the meaning of words as devoid of any deeper significance, I will ask you to regard this question as merely a bond by means of which I have found it convenient to unite what I have to say on the fundamental conceptions and methods of what, with or without definition, we all of us agree to call mathematics.

The old idea that mathematics is the science of quantity, or that it is the science of space and number, or indeed that it can be characterized by any enumeration of several more or less heterogeneous objects of study, has pretty well passed away among those mathematicians who have given any thought to 
the question of what mathematics really is. Such definitions, which might have been intelligently defended at the beginning of the nineteenth century, became obviously inadequate as subjects like projective geometry, the algebra of logic, and the theory of abstract groups were developed; for none of these has any necessary relation to quantity (at least in any ordinary understanding of that word), and the last two have no relation to space. It is true that such examples have had little effect on the more or less orthodox followers of Kant, who regard mathematics as concerned with those conceptions which are obtained by direct intuition of time and space without the aid of empirical observation. This view seems to have been held by such eminent mathematicians as Hamilton and DeMorgan ; and it is a very difficult position to refute, resting as it does on a purely metaphysical foundation which regards it as certain that we can evolve out of our inner consciousness the properties of time and space. According to this view the idea of quantity is to be deduced from these intuitions ; but one of the facts most vividly brought home to pure mathematicians during the last half century is the fatal weakness of intuition when taken as the logical source of our knowledge of number and quantity.*

The objects of mathematical study, even when we confine our attention to what is ordinarily regarded as pure mathematics are, then, of the most varied description; so that, in order to reach a satisfactory conclusion as to what really characterizes mathematics, one of two methods is open to us. On the one hand we may seek some hidden resemblance in the various objects of mathematical investigation, and having found an aspect common to them all we may fix on this as the one true object of mathematical study. Or, on the other hand, we may abandon the attempt to characterize mathematics by means of its objects of study, and seek in its methods its distinguishing characteristic. Finally there is the possibility of our combining these two points of view. The first of these methods is that of Kempe, the second will lead us to the definition of Benjamin Peirce, while the third has recently been elaborated at great length by Russell. Other mathematicians have naturally followed out more or less consistently the same ideas, but I shall nevertheless take the liberty of using the names

\footnotetext{
* I refer here to such facts as that there exist continuous functions without derivatives, whereas the direct untutored intuition of space would lead anyone to believe that every continuous curve has tangents.
} 
Kempe, Peirce, and Russell as convenient designations for these three points of view. These different methods of approaching the question lead finally to results which, without being identical, still stand in the most intimate relation to one another, as we shall now see. Let us begin with the second method.

\section{Peirce's Definition.}

More than a third of a century ago Benjamin Peirce wrote:* Mathematics is the science which draws necessary conclusions. According to this view there is a mathematical element involved in every enquiry in which exact reasoning is used. Thus, for instance, $\nmid$ a jury listening to the attempt of the counsel for the prisoner to prove an alibi in a criminal case might reason as follows. "If the witnesses are telling the truth when they say that the prisoner was in St. Louis at the moment the crime was committed in Chicago, and if it is true that a person cannot be in two places at the same time, it follows that the prisoner was not in Chicago when the crime was committed." This, according to Peirce, is a bit of mathematics; while the further reasoning by which the jury would decide whether or not to believe the witnesses, and the reasoning (if they thought any necessary) by which they would satisfy themselves that a person cannot be in two places at once, would be inductive reasoning which can give merely a high degree of probability to the conclusion, but never certainty. This mathematical element may be, as the example just given shows, so slight as not to be worth noticing from a practical point of view. This is almost always the case in the transactions of daily life and in the observational sciences. If, however, we turn to such subjects as chemistry and mineralogy, we find the mathematical element of considerable importance, though still subordinate. In physics and astronomy its importance is much greater. Finally in geometry, to mention only one other science, the mathematical element predominates to such an extent that this science has been commonly rated a branch of pure mathematics, whereas, according to Peirce, it is as much a branch of applied mathematics as is, for instance, mathematical physics.

It is clear from what has just been said that, from Peirce's

\footnotetext{
* Linear associative algebra. Lithographed 1870. Reprinted in the American Journal of Mathematics, vol. 4.

†This illustration was suggested by the remarks by J. Richard: Sur la philosophie des mathématiques, Paris, Gauthier-Villars, 1903, p. 50.
} 
point of view, mathematics does not necessarily concern itself with quantitative relations, and that any subject becomes capable of mathematical treatment as soon as it has secured data from which important consequences can be drawn by exact reasoning. Thus, for example, even though psychologists be right when they assure us that sensations and the other objects with which they have to deal cannot be measured, we need still not necessarily despair of one day seeing a mathematical psychology, just as we already have a mathematical logic.

I have said enough, I think, to show what relation Peirce's conception of mathematics has to the applications. Let us then turn to the definition itself and examine it a little more closely. You have doubtless already noticed that the phrase: "the science which draws necessary conclusions" contains a word which is very much in need of elucidation. What is a necessary conclusion? Some of you will perhaps think that the conception here involved is one about which, in a concrete case at least, there can be no practical diversity of opinion among men with well trained minds; and in fact when I spoke a few minutes ago about the reasoning of the jurymen when listening to the lawyer trying to prove an alibi, I assumed tacitly that this is so. If this really were the case, no further discussion would be necessary, for it is not my purpose to enter into any purely philosophical speculations. But unfortunately we cannot dismiss the matter in this way; for it has happened not infrequently that the most eminent men, including mathematicians, have differed as to whether a given piece of reasoning was exact or not; and, what is worse, modes of reasoning which seem absolutely conclusive to one generation no longer satisfy the next, as is shown by the way in which the greatest mathematicians of the eighteenth century used geometric intuition as a means of drawing what they regarded as necessary conclusions.*

I do not wish here to raise the question whether there is such a thing as absolute logical rigor, or whether this whole concep-

* All writers on elementary geometry from Euclid down almost to the close of the nineteenth century use intuition freely, though usually unconsciously, in obtaining results which they are unable to deduce from their axioms. The first few demonstrations of Euclid are criticised from this point of view by Russell in his Principles of Mathematics, vol. 1, p. 404-407-Gauss's first proof (1799) that every algebraic equation has a root gives a striking example of the use of intuition in what was intended as an absolutely rigorous proof by one of the greatest and at the same time most critical mathematical minds the world has ever seen. 
tion of logical rigor is a purely psychological one bound to change with changes in the human mind. I content myself with expressing the belief, which I will try to justify a little more fully in a moment, that as we never have found an immutable standard of logical rigor in the past, so we are not likely to find it in the future. However this may be, so much we can say with tolerable confidence, as past experience shows, that no reasoning which claims to be exact can make any use of intuition, but that it must proceed from definitely and completely stated premises according to certain principles of formal logic. It is right here that modern mathematicians break sharply with the tradition of a priori synthetic judgments (that is, conclusions drawn from intuition) which, according to Kant, form an essential part of mathematical reasoning.

If then we agree that "necessary conclusions" must, in the present state of human knowledge, mean conclusions drawn according to certain logical principles from definitely and completely stated premises, we must face the question as to what these principles shall be. Here, fortunately, the mathematical logicians from Boole down to C. S. Peirce, Schröder, and Peano have prepared the field so well that of late years Peano and his followers * have been able to make a rather short list of logical conceptions and principles upon which it would seem that all exact reasoning depends. $\dagger$ We must remember, however, when we are tempted to put implicit confidence in certain fundamental logical principles, that, owing to their extreme generality and abstractness, no very great weight can be attached to the mere fact that these principles appeal to us as obviously true; for, as I have said, other modes of reasoning which are now universally recognized as faulty have appealed in just this way to the greatest minds of the past. Such confidence as we feel must, I think, come from the fact that those modes of reasoning which we trust have withstood the test of use in an immense number of cases and in very many fields. This is the severest test to which any theory can be put, and if it does not break down under it we may feel the greatest confidence that, at least in cognate fields, it will prove serviceable. But we can never be sure. The accepted modes of exact reasoning may any day lead to a contradiction which would show that

\footnotetext{
* And, independently, Frege.

+ It is not intended to assert that a single list has been fixed upon. Different writers naturally use different lists.
} 
what we regard as universally applicable principles are in reality applicable only under certain restrictions. *

To show that the danger which $I$ here point out is not a purely fanciful one, it is sufficient to refer to a very recent example. Independently of one another Frege and Russell have built up the theory of arithmetic from its logical foundations. Each starts with a definite list of apparently self-evident logical principles, and builds up a seemingly flawless theory. Then Russell discovers that his logical principles when applied to a very general kind of logical class lead to an absurdity; and both Frege and Russell have to admit that something is wrong with the foundations which looked so secure. Now there is no doubt that these logical foundations will be somehow recast to meet this difficulty, and that they will then be stronger than ever before. $\dagger$ But who shall say that the same thing will not happen again?

It is commonly considered that mathematics owes its certainty to its reliance on the immutable principles of formal logic. This, as we have seen, is only half the truth imperfectly expressed. The other half would be that the principles of formal logic owe such degree of permanence as they have largely to the fact that they have been tempered by long and varied use by mathematicians. "A vicious circle!" you will perhaps say. I should rather describe it as an example of the process known to mathematicians as the method of successive approximations. Let us hope that in this case it is really a convergent process, as it has every appearance of being.

But to return to Peirce's definition. From what are these necessary conclusions to be drawn? The answer clearly implied is : from any premises sufficiently precise to make it possible to draw necessary conclusions from them. In geometry, for instance, we have a large number of intuitions and fixed

* If the view which I here maintain is correct it follows that if the term 'absolute logical rigor' has a meaning, and if we should some time arrive at this absolute standard, the only indication we should ever have of the fact would be that for a long period, several thousand years let us say, the logical principles in question had stood the test of use. But this state of affairs might equally well mean that during that time the human mind had degenerated, at least with regard to some of its functions. Consider for instance the twenty centuries following Euclid when, without doubt, the high tide of exact thinking attained during Euclid's generation had receded.

+ Cf. Poincaré's view in La science et l'hypothèse, p. 179, according to which a theory never renders a greater service to science than when it breaks down. 
beliefs concerning the nature of space : it is homogeneous and isotropic, infinite in extent in every direction, etc.; but none of these ideas, however clearly defined they may at first sight seem to be, gives any hold for exact reasoning. This was clearly perceived by Euclid, who therefore proceeded to lay down a list of axioms and postulates, that is specific facts which he assumes to be true, and from which it was his object to deduce all geometric propositions. That his success here was not complete is now well known, for he frequently assumes unconsciously further data which he derives from intuition; but his attempt was a monumental one.

\section{The Abstract Nature of Mathematics.}

Now a further self-evident point, but one to which attention seems to have been drawn only during the last few years, is this : since we are to make no use of intuition, but only of a certain number of explicitly stated premises, it is not necessary that we should have any idea what the nature of the objects and relations involved in these premises is.* I will try to make this clear by a simple example. In plane geometry we have to consider, among other things, points and straight lines. A point may have a peculiar relation to a straight line which we express by the words : the point lies on the line. Now one of the fundamental facts of plane geometry is that two points determine a line, that is if two points are given, there exists one and only one line on which both points lie. All the facts that I have just stated correspond to clear intuitions. Let us, however, eliminate our intuition of what is meant by a point, a line, a point lying on a line. A slight change of language will make it easy for us to do this. Instead of points and lines, let us speak of two different kinds of objects, say $A$-objects and $B$-objects; and instead of saying that a point lies on a line we will simply say that an $A$-object bears a certain relation $R$ to a $B$-object. Then the fact that two points determine a line will be expressed by saying: If any two $A$-objects are given, there exists one and only one $B$-object to which they both bear the relation $R$. This statement, while it does not force on us any specific intuitions, will serve as a basis for mathematical reason-

\footnotetext{
* This was essentially Kempe's point of view in the papers to be referred to presently. In the geometric example which follows it was clearly brought out by $\mathrm{H}$. Wiener : Jahresbericht d. deutschen Mathematiker-Vereinigung, vol. 1 (1891), p. 45.
} 
ing * just as well as the more familiar statement where the terms points and lines are used. But more than this. Our $A$-objects, our $B$-objects and our relation $R$ may be given an interpretation, if we choose, very different from that we had at first intended.

We may, for instance, regard the $A$-objects as the straight lines in a plane, the $B$-objects as the points in the same plane (either finite or at infinity), and when an $A$-object stands in the relation $R$ to a $B$-object this may be taken to mean that the line passes through the point. Our statement would then become : Any two lines being given, there exists one and only one point through which they both pass. Or we may regard the $A$-objects as the men in a certain community, the $B$-objects as the women, and the relation of an $A$-object to a $B$-object as friendship. Then our statement would be : In this community any two men have one, and only one, woman friend in common.

These examples are, $I$ think, sufficient to show what is meant when I say that we are not concerned in mathematics with the nature of the objects and relations involved in our premises, except in so far as their nature is exhibited in the premises themselves. Accordingly mathematicians of a critical turn of mind, during the last few years, have adopted more and more a purely nominalistic attitude towards the objects and relations involved in mathematical investigation. This is, of course, not the crude mixture of nominalism and empiricism of the philosopher Hobbes, whose claim to mathematical fame, it may be said in passing, is that of a circle squarer. $\dagger$ The nominalism of the present day mathematician consists in treating the objects of his investigation and the relations between them as mere symbols. He then states his propositions, in effect, in the following form : If there exist any objects in the physical or mental world with relations among themselves which satisfy the conditions which I have laid down for my symbols, then such and such facts will be true concerning them.

It will be seen that, according to Peirce's view, the mathematician as such is in no wise concerned with the source of his premises or with their harmony or lack of harmony with any part of the external world. He does not even assert that any

\footnotetext{
* In conjunction of course with further postulates with which we need not here concern ourselves.

$\dagger$ Hobbes practically obtains as the ratio of a circumference to its diameter the value $\sqrt{10}$. Cf. for instance Molesworth's edition of Hobbes's English Works, vol. 7, p. 431.
} 
objects really exist which correspond to his symbols. Mathematics may therefore be truly said to be the most abstract of all sciences, since it does not deal directly with reality.*

This, then, is Peirce's definition of mathematics. Its advantages in the direction of unifying our conception of mathematics and of assigning to it a definite place among the other sciences are clear. What are its disadvantages? I can see only two. First that, as has been already remarked, the idea of drawing necessary conclusions is a slightly vague and shifting one. Secondly that it lays exclusive stress on the rigorous logical element in mathematics and ignores the intuitional and other non-rigorous tendencies which form an important element in the great bulk of mathematical work, concerning which I shall speak in greater detail later.

\section{Geometry un Experimental Science.}

Some of you will also regard it as an objection that there are subjects which have almost universally been regarded as branches of mathematics but are excluded by this definition. A striking example of this is geometry, I mean the science of the actual space we live in ; for though geometry is, according to Peirce's definition, preëminently a mathematical science, it is not exclusively so. Until a system of axioms is established mathematics cannot begin its work. Moreover the actual perception of spacial relations, not merely in simple cases but in the appreciation of complicated theorems, is an essential element in geometry which has no relation to mathematics as Peirce understands the term. The same is true, to a considerable extent, of such subjects as mechanical drawing and model making, which involve, besides small amounts of physics and mathematics, mainly non-mathematical geometry. Moreover, although the mathematical method is the traditional one for arriving at the truth concerning geometric facts, it is not the only one. Direct appeal to the intuition is often a short and fairly safe cut to geometric results; and on the other hand experiments may be used in geometry, just as they are used every day in physics, to test the truth of a proposition or to determine the value of some geometric magnitude. $\dagger$

\footnotetext{
* Cf. the very interesting remarks along this line of C. S. Peirce in The Monist, vol. 7, pp. 23-24.

$\dagger$ I am thinking of measurements and observations made on accurately constructed drawings and models. A famous example is Galileo's determination of the area of a cycloid by cutting out a cycloid from a metallic sheet and weighing it.
} 
We must, then, admit, if we hold to Peirce's view, that there is an independent science of geometry just as there is an independent science of physics, and that either of these may be treated by mathematical methods. Thus geometry becomes the simplest of the natural sciences, and its axioms are of the nature of physical laws, to be tested by experience and to be regarded as true only within the limits of error of observation. This view, while it has not yet gained universal recognition, should, I believe, prevail, and geometry be recognized as a science independent of mathematics, just as psychology is gradually being recognized as an independent science and not as a branch of philosophy.

The view here set forth, according to which geometry is an experimental science like physics or chemistry, has been held ever since Gauss's time by almost all the leading mathematicians who have been conversant with non-euclidean geometry.* Recently, however, Poincare has thrown the weight of his great authority against this view, $\nmid$ claiming that the experiments by which it is sought to test the truth of geometric axioms are really not geometric experiments at all but physical ones, and that any failure of these experiments to agree with the ordinary geometric axioms could be explained by the inaccuracy of the physical laws ordinarily assumed. There is undoubtedly an important element of truth here. Every experiment depends for its results not merely on the law we wish to test but also on other laws which for the moment we assume to be true. But, if we prefer, we may, in many cases, assume as true the law we were before testing and our experiment will then serve to test some of the remaining laws. If, then, we choose to stick to the ordinary euclidean axioms of geometry in spite of what any future experiments may possibly show, we can do so, but at the cost, perhaps, of our present simple physical laws, not merely in one branch of physics but in several. Poincarés view $\$$ is that it will always be expedient to preserve simple geometric laws at all costs, an opinion for which I fail to see sufficient reason.

\footnotetext{
* Gauss, Riemann, Helmholtz are the names which will carry perhaps the greatest weight.

† Cf. La soience et l'hypothèse. Paris, 1903.

$\ddagger$ L. c., chapter 5 . In particular p. 93 .
} 


\section{Kempe's Definition.}

Let us now turn from Peirce's method of defining mathemathics to Kempe's, which, however, I shall present to you in a somewhat modified form.* The point of view adopted here is to try to define mathematics, as other sciences are defined, by describing the objects with which it deals. The diversity of the objects with which mathematics is ordinarily supposed to deal being so great, the first step must be to divest them of what is unessential for the mathematical treatment, and to try in this way to discover their common and characteristic element.

The first point on which Kempe insists is that the objects of mathematical discussion, whether they be the points and lines of geometry, the numbers real or complex of algebra or analysis, the elements of groups or anything else, are always individuals, infinite in number perhaps, but still distinct individuals. In a particular mathematical investigation we may, and usually do, have several different kinds of individuals; as for instance, in elementary plane geometry points, straight lines, and circles. Furthermore we have to deal with certain relations of these objects to one another. For instance, in the example just cited, a given point may or may not lie on a given line; a given line may or may not touch a given circle; three or more points may or may not be collinear, etc. This example shows how in a single mathematical problem a large number of relations may be involved, relations some of which connect two objects, others three, etc. Moreover these relations may connect like or they may connect unlike objects; and finally the order in which the objects are taken is not by any means immaterial in general, as is shown by the relation between three points which states that the third is collinear with and lies between the first two.

But even this is not all ; for, besides these objects and relations of various kinds, we often have operations by which objects can be combined to yield another object, as, for instance, addition or multiplication of numbers. Here the objects combined and the resulting object are all of the same kind, but this is by no means necessary. We may, for instance, con-

\footnotetext{
* Kempe has set forth his ideas in rather popular form in the Proceedings of the London Mathematical Society, vol. 26 (1894), p. 5 ; and in Nature, vol. $43(1890)$, p. 156, where references to his more technical writings will be found.
} 
sider the operation of combining two points and getting the perpendicular bisector of the line connecting them; or we may combine a point and a line and get the perpendicular dropped from the point on the line.

These few examples show how diverse the relations and operations, as well as the objects of mathematics seem at first sight to be. Out of this apparent diversity it is not difficult to obtain a very great uniformity by simply restating the facts in a little different language. We shall find it convenient to indicate that the objects $a, b, c, \cdots$, taken in the order named, satisfy a relation $R$ by simply writing $R(a, b, c, \cdots)$, where it should be understood that among the objects $a, b, c, \cdots$ the same object may occur a number of times. On the other hand if two objects $a$ and $b$ are combined to yield a third object $c$ we may write $a \circ b=c$,* where the symbol o is characteristic of the special operation with which we are concerned.

Let us first notice that the equation $a \circ b=c$ denotes merely that the three objects $a, b, c$ bear a certain relation to one another, say $R(a, b, c)$. In other words the idea of an operation or law of combination between the objects we deal with, however convenient and useful it may be as a matter of notation, is essentially merely a way of expressing the fact that the objects combined bear a certain relation to the object resulting from their combination. Accordingly, in a purely abstract discussion like the present, where questions of practical convenience are not involved, we need not consider such rules of combination. $\dagger$

Furthermore it is easy to see that when we speak of objects of different kinds, as for instance the points and lines of geom-

\footnotetext{
* I speak here merely of dyadic operations, - i. e , of operations by which two objects are combined to yield a third, - these being by far the most important as well as the simplest. What is said, however, obviously applies to operations by which any number of objects are combined.

$\dagger$ Even from the point of view of the technical mathematician it may sometimes be desirable to adopt the point of view of a relation rather than that of an operation. This is seen, for instance, in laying down a system of postulates for the theory of abstract groups (cf., for example, Huntington, Bulletin, June, 1902), where the postulate:

If $a$ and $b$ belong to the class, $a \circ b$ belongs to the class, which in this form looks indecomposable, immediately breaks up, when stated in the relational form, into the following two :

1. If $a$ and $b$ belong to the class, there exists an element $c$ of the class such that $R(a, b, c)$. $c=d$.

2. If $a, b, c, d$ belong to the class, and if $R(a, b, c)$ and $R(a, b, d)$, then
} 
etry, we are introducing a notion which can very readily be expressed in our relational notation. For this purpose we need merely to introduce a further relation which is satisfied by two or more objects when and only when they are of the same " kind."

Let us turn finally to the relations themselves. It is customary to distinguish here between dyadic relations, triadic relations, etc., according as the relation in question connects two objects, three objects, etc. There are, however, relations which may connect any number of objects, as, for instance, the relation of collinearity which may hold between any number of points. Any relation holds for certain ordered groups of objects but not for others, and it is in no way necessary for us to fix our attention on the fact, if it be true, that the number of objects in all the groups for which a particular relation holds is the same. This is the point of view we shall adopt, and we shall relegate the property that a relation is dyadic, triadic, etc., to the background along with the various other properties relations may have,* all of which must be taken account of in the proper place.

We are thus concerned in any mathematical investigation from our present point of view with just two conceptions : first a set, or as the logicans say, a class of objects $a, b, c, \cdots$; and secondly a class of relations $R, S, T, \ldots$. We may suppose these objects divested of any qualitative, quantitative, special or other attributes which they may have had, and regard them merely as satisfying or not satisfying the relations in question, where, again, we are wholly indifferent to the nature which these relations originally had. And now we are in a position to state what $I$ conceive to be really the essential point in Kempe's definition of mathematics, although I have omitted one of the points on which he insists most strongly, $\dagger$ by saying :

If we have a certain class of objects and a certain class of relations, and if the only questions which we investigate are whether ordered groups of these objects do or do not satisfy the relations, the results of the investigation are called mathematics.

\footnotetext{
* For instance the property of symmetry. A relation is said to be symmetric if it holds or fails to hold independently of the order in which the objects are taken.

$\dagger$ Namely that the only relation that need be considered is that of being "indistinguishable," i. e., a symmetric and transitive relation between two groups of objects.
} 
It is convenient to have a term to designate a class of objects associated with a class of relations between these objects. Such an aggregate we will speak of as a mathematical system. If now we have two different mathematical systems, and if a oneto-one correspondence can be set up between the two classes of objects, and also between the two classes of relations in such a way that whenever a certain ordered set of objects of the first system satisfies a relation of that system, the set consisting of the corresponding objects of the second system satisfies the corresponding relation of that system, and vice versa, then it is clear that the two systems are, from our present point of view, mathematically equivalent, however different the nature of the objects and relations may be in the two cases.* To use a technical term, the two systems are simply isomorphic. $\dagger$

It will be noticed that in the definition of mathematics just given nothing is said as to the method by which we are to ascertain whether or not a given relation holds between the objects of a given set. The method used may be a purely empirical one, or it may be partly or wholly deductive. Thus, to take a very simple case, suppose our class of objects to consist of a large number of points in a plane, and suppose the only relation between them with which we are concerned is that of collinearity. Then, if the points are given us by being marked in ink on a piece of white paper, we can begin by taking three pins, sticking them into the paper at three of the points; then, by sighting along them, we can determine whether or not these points are collinear. We can do the same with other groups of three points, then with all groups of four points, etc. The same result can be obtained with much less labor if we make use of certain simple properties which the relation of collinearity satisfies, properties which are expressed by such propositions as :

\footnotetext{
* The point of view here brought out, including the term isomorphism, was first developed in a special case - the theory of groups.

+ Inasmuch as the relations in a mathematical system are themselves objects, we may, if we choose, take our class of objects so as to include these relations as well as what we called objects before, some of which, we may remark in passing, may themselves be relations. Looked at from this point of view we need one additional relation which is now the only one which we explicitly call a relation. If we denote this relation by enclosing the objects which satisfy it in parentheses, then if the relation denoted before by $R(a, b)$ is satisfied we should now write $(R, a, b)$, whereas wo should not have $(a, R, b)(S, R, a, b)$, etc. Thus we see that any mathematical system may be regarded as consisting of a class of objects and a single relation between them.
} 
$R(a, b, c)$ implies $R(b, a, c)$,

$R(a, b, c, d)$ implies $R(a, b, c)$,

$R(a, b, c)$ and $R(a, b, d)$ together imply $R(a, b, c, d)$, etc.

By means of a small number of propositions of this sort it is easy to show that no empirical observations as to the collinearity of groups of more than three points need be made, and that it may not be necessary to examine even all groups of three points. Having made this relatively small number of observations, the remaining results would be obtained deductively. Finally, we may suppose the points given by their coördinates, in which case the complete answer to our question may be obtained by the purely deductive method of analytic geometry.

According to the modified form of Kempe's definition which I have just stated, mathematics is not necessarily a deductive science. This view, while not in accord with the prevailing ideas of mathematicians, undoubtedly has its advantages as well as its dangers. The non-deductive processes, of which I shall have more to say presently, play too important a part in the life of mathematics to be ignored, and the definition just given has the merit of not excluding them. It would seem, however, that the definition in the form just given is too broad. It would include, for instance, the determination by experimental methods of what pairs of chemical compounds of the known elements react on one another when mixed under given conditions.

\section{Axioms and Postulates. Existence Theorems.}

If, however, we restrict ourselves to exact or déductive mathematics, it will be seen that Kempe's definition becomes coëxtensive with Peirce's. Here, in order to have a startingpoint for deductive reasoning, we must assume a certain number of facts or primitive propositions concerning any mathematical system we wish to study, of which all other propositions will be necessary consequences.* We touch here on a subject whose origin goes back to Euclid and which has of late years received great development, primarily at the hands of Italian mathematicians. $\uparrow$

\footnotetext{
* These primitive propositions may be spoken of as axioms or postulates, according to the point of view we wish to take concerning their source, the word axiom, which has been much misused of late, indicating an intuitional or empirical source.

† Peano, Pieri, Padoa, Burali-Forti. We may mention here also Hilbert, who, apparently without knowing of the important work of his Italian predecessors, has also done valuable work along these lines.
} 
It is important for us to notice at this point that not merely these primitive propositions but all the propositions of mathematics may be divided into two great classes. On the one hand, we have propositions which state that certain specified objects satisfy certain specified relations. On the other hand are the existence theorems which state that there exist objects satisfying, along with certain specified objects, certain specified relations.* These two classes of propositions are well known to logicians and are designated by them universal and particular propositions respectively. $\dagger$ It is only during the last fifty years or so that mathematicians have become conscious of the fundamental importance in their science of existence theorems which until then they had frequently assumed tacitly as they needed them, without always being conscious of what they were doing.

It is sometimes held by non-mathematicians that if mathematics were really a purely deductive science, it could not have gained anything like the extent which it has without losing itself in trivialities and becoming, as Poincaré puts it, a vast tautology.t This view would doubtless be correct if all primitive propositions were universal propositions. One of the most characteristic features of mathematical reasoning, however, is the use which it makes of auxiliary elements. I refer to the auxiliary points and lines in proofs by elementary geometry, the quantities formed by combining in various ways the numbers which enter into the theorems to be proved in algebra, etc. Without the use of such auxiliary elements mathematicians would be incapable of advancing a step ; and whenever we make use of such an element in a proof, we are in reality using an existence-theorem. $\S$ These existence theorems need

* Or we might conceivably have existence theorems which state that there exist relations which are sati-fied hy certain specified objects; or these two kinds of existence theorems might be combined. If we take the point of view explained in the footnote on p. 128, all existence theorems will be of the type mentioned in the text.

+ "All men are mortals" is a standard example of a universal proposition; while as an illustration of a particular proposition is often given : "Some men are Greeks" That this is really an existence theorem is seen more clearly when we state it in the form: "There exists at least one man who is a Greek."

$¥$ Cf. La Science et l'hypothèse, p. 10.

$\$$ Even when in algebra we consider the sum of two numbers $a+b$, we are using the existence theorem which says that, any two numbers $a$ and $b$ being given, there exists a number $c$ which stands to them in the relation which we indicate in ordinary language by saying that $c$ is the sum of $a$ and $b$. 
not, to be sure, be among the primitive propositions; but if not they must be deduced from primitive propositions some of which are existence-theorems, for it is clear that an existence theorem cannot be deduced from universal propositions alone.* Thus it may fairly be said that existence theorems form the vital principle of mathematics, but these in turn, it must be remembered, would be impotent without the material basis of universal propositions to work upon.

\section{Russell's Definition.}

We have so far arrived at the view that exact mathematics is the study by deductive methods of what we have called a mathematical system, that is a class of objects and a class of relations between them. If we elaborate this position in two directions we shall reach the standpoint of Russell. $\dagger$

In the first place Russell makes precise the term deductive method by laying down explicitly a list of logical conceptions, and principles which alone are to be used; and secondly he is not willing that mathematics-at least pure mathematicsshould be, as Peirce would have it, a science of hypotheses which is not concerned with the truth or falsity of its premises. $\mathrm{He}$ insists, on the contrary, that no mathematical system, to use again the technical term introduced above, be studied in pure mathematics whose existence cannot be established from the logical principles on which all mathematics is based. Inasmuch as the development of mathematics during the last fifty years has shown that the existence of most, if not all the mathematical systems which have proved to be important can be deduced when once the existence of positive integers is granted, the point about which interest must centre here is the proof, which Russell attempts, of the existence of this latter system. $\S$

* The power which resides in the method of mathematical induction, socalled, comes from the fact that this method depends on an existence theorem. It is, however, not the only fertile principle in mathematics as Poincaré would have us believe (cf. La science et l'hypothèse). In fact there are great branches of mathematics like elementary geometry, in which it takes little or no part.

†The Principles of Mathematics, Cambridge, England, 1903.

$\ddagger$ In the formal definition of mathematics at the beginning of the book this is not stated or in any way implied; and yet it comes out so clearly throughout the book that this is a point of view which the author regards as essential, that I have not hesitated to include it as a part of his definition.

8. Cf. also Burali-Forti, Congrès internationale de philosophie. Paris, vol. 3 , p. 289. 
This proof will necessarily require that, among the logical principles assumed, existence theorems be found. Such theorems do not seem to be explicitly stated by Russell, the existence theorems which make their appearance further on being evolved out of somewhat vague philosophical reasoning. There are also other reasons, into which I cannot enter here, why I am not able to regard the attempt made in this direction by Russell as completely successful.* Nevertheless, in view of the fact that the system of finite positive integers is necessary in almost all branches of mathematics (we cannot speak of a triangle or a hexagon without having the numbers three and six at our disposal), it seems extremely desirable that the system of logical principles which we lay at the foundation of all mathematics be assumed, if possible, broad enough so that the existence of positive integers - at least finite integers - follows from it; and there seems little doubt that this can be done in a satisfactory manner. When this has been done we shall perhaps be able to regard, with Russell, pure mathematics as consisting exclusively of deductions by "logical principles from logical principles."

\section{The Non-Deductive Elements in Mathematics.}

I fear that many of you will think that what I have been saying is of an extremely one-sided character, for I have insisted merely on the rigidly deductive form of reasoning used and the purely abstract character of the objects considered in mathematics. These, to the great majority of mathematicians, are only the dry bones of the science. Or, to change the simile, it may perhaps be said that instead of inviting you to a feast I have merely shown you the empty dishes and explained how the feast would be served if only the dishes were filled. $\dagger$ I fully agree with this opinion, and can only plead in excuse that my subject was the fundamental conceptions and methods of mathematics, not the infinite variety of detail and application which give our science its real vitality. In fact I should like to subscribe most heartily to the view that in mathematics, as elsewhere, the

* Cf. a paper read by the present writer before the meeting of the American Mathematical Society three days before the present address was delivered, in which this question was discussed. Russell's unequivocal repudiation of nominalism in mathematics seems to me a serious if not an insurmountable barrier to progress.

$\uparrow$ Notice that just as the empty dishes could be filled by a great variety of viands, so the empty symbols of mathematics can be given meanings of the most varied sorts. 
discussion of such fundamental matters derives its interest mainly from the importance of the theory of which they are the so-called foundations.* I like to look at mathematics almost more as an art than as a science; for the activity of the mathematician, constantly creating as he is, guided though not controlled by the external world of the senses, bears a resemblance, not fanciful I believe but real, to the activity of an artist, of a painter let us say. Rigorous deductive reasoning on the part of the mathematician may be likened here to technical skill in drawing on the part of the painter. Just as no one can become a good painter without a certain amount of this skill, so no one can become a mathematician without the power to reason accurately up to a certain point. Yet these qualities, fundamental though they are, do not make a painter or a mathematician worthy of the name, nor indeed are they the most important factors in the case. Other qualities of a far more subtle sort, chief among which in both cases is imagination, go to the making of the good artist or good mathematician. I must content myself by merely recalling to you this somewhat vague and difficult though interesting field of speculation which arises when we attempt to attach value to mathematical work, a field which is familiar enough to us all in the analogous case of artistic or literary criticism.

We are in the habit of speaking of logical rigor and the consideration of axioms and postulates as the foundations on which the superb structure of modern mathematics rests; and it is often a matter of wonder how such a great edifice can rest securely on such a small foundation. Moreover these foundations have not always seemed so secure as they do at present. During the first half of the nineteenth century certain mathematicians of a critical turn of mind - Cauchy, Abel, Weierstrass, to mention the greatest of them - perceived to their dismay that these foundations were not sound, and some of the best efforts of their lives were devoted to strengthening and improving them. And yet I doubt whether the great results of mathematics seemed less certain to any of them because of the weakness they perceived in the foundations on which these

* Cf. the following remark by Study, Jahresbericht der deutschen Mathematiker-Vereinigung, vol. 11 (1902), p. 313 :

"So wertvoll auch Untersuchungen über die systematische Stellung der mathematischen Grundbegriffe sind . . . wertvoller ist doch noch der materielle Inhalt der einzelnen Disciplinen, um dessentwillen allein ja derartige Untersuchungen überhaupt Zweck haben. . . ." 
results are built up. The fact is that what we call mathematical rigor is merely one of the foundation stones of the science; an important and essential one surely, yet not the only thing upon which we can rely. A science which has developed along such broad lines as mathematics, with such numerous relations of its parts both to one another and to other sciences, could not long contain serious error without detection. This explains how, again and again, it has come about, that the most important mathematical developments have taken place by methods which cannot be wholly justified by our present canons of mathematical rigor, the logical "foundation" having been supplied only long after the superstructure had been raised. A discussion and analysis of the non-deductive methods which the creative mathematician really uses would be both interesting and instructive. Here I must content myself with the enumeration of a few of them.

First and foremost there is the use of intuition, whether geometric, mechanical, or physical. The great service which this method has rendered and is still rendering to mathematics both pure and applied is so well known that a mere mention is sufficient.

Then there is the method of experiment; not merely the physical experiments of the laboratory or the geometric experiments I had occasion to speak of a few minutes ago, but also arithmetical experiments, numerous examples of which are found in the theory of numbers and in analysis. The mathematicians of the past frequently used this method in their printed works. That this is now seldom done must not be taken to indicate that the method itself is not used as much as ever.

Closely allied to this method of experiment is the method of analogy which assumes that something true of a considerable number of cases will probably be true in analogous cases. This is, of course, nothing but the ordinary method of induction. But in mathematics induction may be employed not merely in connection with the experimental method, but also to extend results won by deductive methods to other analogous cases. This use of induction has often been unconscious and sometimes overbold, as, for instance, when the operations of ordinary algebra were extended without scruple to infinite series.

Finally there is what may perhaps be called the method of optimism which leads us either wilfully or instinctively to shut 
our eyes to the possibility of evil. Thus the optimist who treats a problem in algebra or analytic geometry will say, if he stops to reflect on what he is doing: "I know that I have no right to divide by zero; but there are so many other values which the expression by which I am dividing might have that I will assume that the Evil One has not thrown a zero in $\mathrm{my}$ denominator this time." This method, if a proceeding often unconscious can be called a method, has been of great service in the rapid development of many branches of mathematics, though it may well be doubted whether in a subject as highly developed as is ordinary algebra it has not now survived its usefulness.*

While no one of these methods can in any way compare with that of rigorous deductive reasoning as a method upon which to base mathematical results, it would be merely shutting one's eyes to the facts to deny them their place in the life of the mathematical world, not merely of the past but of today. There is now, and there always will be room in the world for good mathematicians of every grade of logical precision. It is almost equally important that the small band whose chief interest lies in accuracy and rigor should not make the mistake of despising the broader though less accurate work of the great mass of their colleagues; as that the latter should not attempt to shake themselves wholly free from the restraint the former would put upon them: The union of these two tendencies in the same individuals, as it was found, for instance, in Gauss and Cauchy, seems the only sure way of avoiding complete estrangement between mathematicians of these two types.

\footnotetext{
* Cf. the very suggestive remarks by Study, Jahresbericht $d$. Deutschen Mathematiker-Vereinigung, vol. 11 (1902), p. 10(), footnote, in which it is pointed out how rigor, in cases of this sort, may not merely serve to increase the correctness of the result, but actually to suggest new fields for mathematical investigation.
} 